\title{
A Two-Phased Guidance Law for Impact Angle Control with Seeker's Field-of-View Limit
}

\author{
Haoqiang Zhang $\mathbb{D},{ }^{1}$ Shengjing Tang $\mathbb{D}^{1},{ }^{1}$ Jie Guo $\mathbb{D},{ }^{1}$ and Wan Zhang $\mathbb{D}^{2}$ \\ ${ }^{1}$ Key Laboratory of Dynamic and Control of Flight Vehicle of the Ministry of Education, School of Aerospace Engineering, \\ Beijing Institute of Technology, Beijing 100081, China \\ ${ }^{2}$ Beijing Electro-Mechanical Engineering Institute, Beijing 100071, China
}

Correspondence should be addressed to Jie Guo; guojie1981@bit.edu.cn

Received 25 July 2017; Revised 27 October 2017; Accepted 12 November 2017; Published 25 March 2018

Academic Editor: Hikmat Asadov

Copyright (c) 2018 Haoqiang Zhang et al. This is an open access article distributed under the Creative Commons Attribution License, which permits unrestricted use, distribution, and reproduction in any medium, provided the original work is properly cited.

\begin{abstract}
A two-phased guidance problem with terminal impact angle constraints and seeker's field-of-view limit is addressed in this paper for a missile against a nonmaneuvering incoming target. From the conventional PN guidance without any constraints, it is found that satisfying the impact angle constraint causes a more curved missile trajectory requiring a large look angle. To avoid the look angle exceeding the seeker's physical limit, a two-phased look angle control guidance scheme with the terminal constraint is introduced. The PN-typed guidance law is designed for each guidance phase with a specific switching condition of line-of-sight. The proposed guidance law is comprised of two types of acceleration commands: the one in the initial phase which aims at controlling the missile's look angle to reach the limit and the other for final phase which is produced by switching the navigation gain. The monotonicity of the line-of-sight angle and look angle is analyzed and proved to support the proposed method. To evaluate the specific navigation gains for both initial and final phases, the scaling coefficient between them is discussed by solving a quadratic equation with respect to the initial navigation gain. To avoid a great abrupt acceleration change at the switching instant, a minimum coefficient is chosen. Extensive simulations are performed to validate the efficiency of the proposed approach.
\end{abstract}

\section{Introduction}

The impact angle control guidance laws (IACGL) have been widely studied for several years [1], for both stationary and moving targets. Practically, the engagement cannot be implemented successfully for a homing missile without the target information from a seeker. The seeker's field-of-view (FOV) is a critical limit for missile engagement, especially when the missile utilizes a seeker with narrow FOV and intercepts a high-speed target with terminal angle constraints $[2,3]$. In such a strict condition, the line-of-sight (LOS) violates the FOV limit more easily. Meanwhile, the missile's detection process might be within a short period of time for moving targets.

A considerable number of previous works focusing on the impact angle guidance used the optimal control theory or the proportional navigation (PN) guidance method. The optimal control theory is mainly used to design those guidance laws in the assumption of linear engagement model. This method is typically applied with a class of cost functions involving the quadratic term of the control input which needs to be minimized [4]. By using the Schwartz inequality, the guidance laws which considered the terminal impact angle constraints (TIAC) were developed with the estimated time-to-go $[5,6]$, especially for a type of linear quadratic optimal control problem. The optimal guidance laws (OGL) for impact angle control which were proposed in $[7,8]$ also paid attention to the other performance of the shaping trajectory, such as the target observability and the acceleration constraints. Because of its efficiency and ease of implementation, $\mathrm{PN}$ guidance law is more popular and widely used [9]. There are also many modified PN-typed laws related to the impact angle control problem. A biased PN (BPN) guidance law was adopted in [10] to control the missile to impact a 
target with terminal angular constraints. This guidance law is a variation of the conventional PN guidance law, which is combined with a supplementary time-varying bias to control the impact angle. Lu et al. [11] introduced a threedimensional guidance law, which was also based on PN with adaptive guidance parameters, to control a hypersonic vehicle in its terminal phase to impact a stationary target. Ratnoo and Ghose [12,13] proposed a two-phased variable navigation gain $\mathrm{PN}$ guidance law for intercepting stationary and moving (nonmaneuvering) targets with all possible impact angles, but not considering the FOV limitation. On the other hand, sliding mode control (SMC) theory becomes more and more popular to be applied to design IACGL. A finite-time convergent sliding mode guidance law with terminal impact angle constraint was presented in [14], which was mainly addressed by the finite-time convergence stability theory. Zhao et al. [15] designed a SMCbased guidance law for an unpowered lifting reentry vehicle against a stationary target, to satisfy the TIAC with high guidance accuracy. Recently, the interception against maneuvering target was proposed based on several new SMC methods, for example, the nonsingular fast sliding mode (NFSM), with consideration of TIAC $[16,17]$.

Furthermore, several works are introduced to design the guidance laws considering the FOV limit as well as the TIAC. Park et al. [18] proposed an optimal impact angle control guidance law with the seeker's FOV limit for missiles with strapdown seekers. By using the optimal control theory, the look angle which was regarded as a new state for inequality constraint was introduced during the homing phase. In [19], a two-phased scheme was developed in which BPN was used to shape the missile trajectory by making the integral value of the bias to reach a certain value at first and then switched to PN in the second phase. The integral value was calculated from initial engagement conditions and desired impact angle. Based on the same strategy of [19], a few of two-phased BPN (TPBPN) guidance laws were proposed in [20-25] for attacking a stationary target. A TPBPN was proposed in [20] based on the bias-shaping method, which can satisfy both the terminal-angle constraint and the FOV limitation to maintain the seeker's lock-on condition. The TPBPN in [21] utilized the unbiased and biased pure proportional navigation laws and applied seeker's FOV maximum value to calculate the bias in the initial phase. A time-varying BPN guidance law was designed by Yang et al. [22] to achieve the angular constraints without violating the look angle. Two time-varying biases were adopted in [22] to keep the seeker's look angle and to ensure the terminal impact angle, respectively. Along with the thoughts of TPBPN and composite guidance law, Tekin and Erer [23] proposed the PN-gain-switched strategy, which can admit the allowed look angle and acceleration values to meet the demand of impact angle and at the same time, have a relative simple structure for implementation because of the PN form. Based on a two-stage PN guidance law, Ratnoo [24] derived a closed-form solution for the choice of navigation gains to satisfy the look angle and impact angle limit. Wen et al. [25] proposed a new guidance parameter design strategy based on the classical time-to-go weighted impact angle optimal guidance law. A robust guidance law which was based on the switching logic was designed in [26] by an additional term and combined the sliding mode control. This kind of guidance problem was also solved by SMC [27] and handled without any additional switching logic. However, since most of these works dealt with stationary targets, the desired impact angle may not be achieved against moving targets when applying these works to homing missiles. A composite guidance scheme was presented considering the case of a nonmaneuvering moving target, which was composed of modified deviated pure pursuit (DPP) with error feedback loop of look angle for initial guidance phases and PN with $N \geq 3$ for final guidance phases [28]. Park et al. [29] addressed the similar guidance problem and strategy in [28] and extended to consider the command limit.

Based on the studies of previous works, this article draws on the experience of PN-gain-switched strategy from [23] to extend the investigated case to a nonmaneuvering moving target. The algorithm procedures and switching logic are introduced to achieve the guidance law proposed in this work. The main contributions of this work are summarized as follows:

(1) We have studied the scaling factor between the different navigation gains of the initial and final guidance phases. The minimum value of this scaling factor is chosen to reduce the abrupt acceleration change at switching instant. Therefore, the specific navigation gain values for both two guidance phases can be calculated according to the scaling factor and the desired impact angle, which is different from $[23,28]$.

(2) Two propositions are presented to prove the monotonicity of the line-of-sight angle and look angle. The switching of guidance phases occurs when a specific LOS angle similar to [28] is satisfied. However, the look angle reaches the FOV limit only at the switching instant, which reduces the required load in initial guidance phase compared with [28].

(3) Different from [20-22], the integral biased term is not needed in the proposed method, which indicates that we do not need to estimate the value of timeto-go. It results in a convenient implementation in real missile model.

\section{Problem Statement}

Consider the planar homing guidance geometry of a missile with narrow FOV against a nonmaneuvering incoming target as shown in Figure 1, where $X_{\mathrm{I}}-O-Y_{\mathrm{I}}$ is a Cartesian inertial reference frame. The relative distance between the missile and the target is $r$. The subscripts $\mathrm{M}$ and $\mathrm{T}$ denote the missile and the target, respectively. The acceleration vector $\mathbf{a}$ is applied perpendicularly to the velocity vector $\mathbf{V} . \gamma, \lambda$, and $\sigma$ represent the flight-path angle, LOS angle, and look angle. Angles are positive in the counterclockwise direction.

As characteristics of the surface-to-surface engagement, the flight-path angle of target equals to $\pi$ which is under the condition of an incoming target. The initial condition 


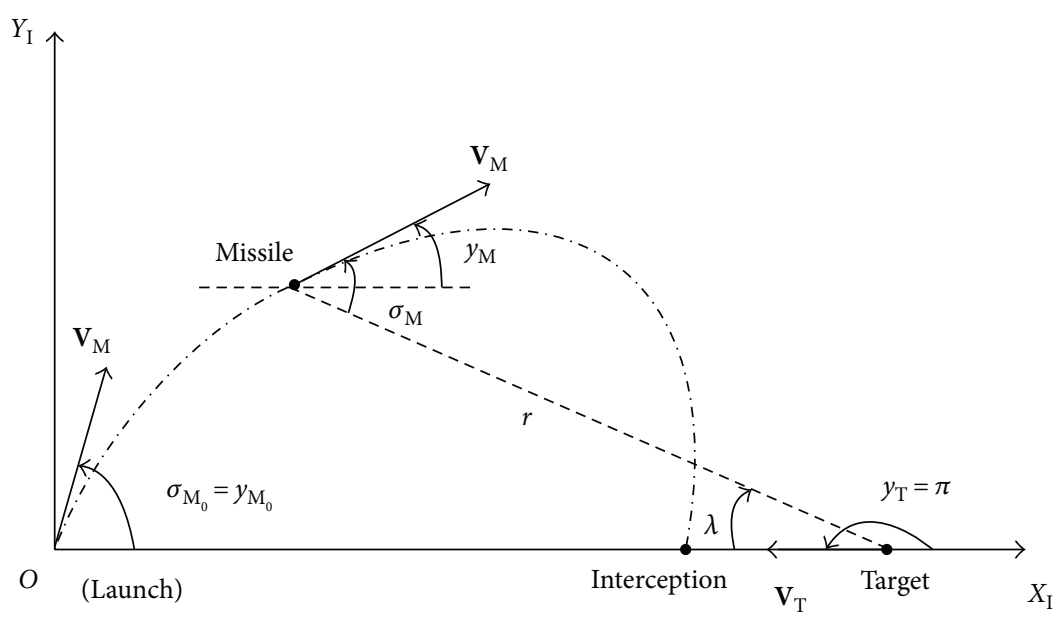

FIGURE 1: Engagement geometry.

on the launch point is also characterized with $\gamma_{\mathrm{M}_{0}}=\sigma_{\mathrm{M}_{0}}$. These conditions imply an approximate head-on case with initial heading errors. The nonlinear equations of motion in this homing problem are given by

$$
\begin{aligned}
\dot{r} & =-\mathbf{V}_{\mathrm{T}} \cos \lambda-\mathbf{V}_{\mathrm{M}} \cos \sigma_{\mathrm{M}}, \\
r \dot{\lambda} & =\mathbf{V}_{\mathrm{T}} \sin \lambda-\mathbf{V}_{\mathrm{M}} \sin \sigma_{\mathrm{M}}, \\
\dot{\gamma}_{\mathrm{M}} & =\frac{\mathbf{a}_{\mathrm{M}}}{\mathbf{V}_{\mathrm{M}}} \\
\sigma_{\mathrm{M}} & =\gamma_{\mathrm{M}}-\lambda,
\end{aligned}
$$

where $\sigma_{\mathrm{M}}$ can be defined as the seeker's look angle in the assumption that the angle of attack (AOA) of the missile is small.

If the missile follows PN guidance law with a navigation gain $N$, the flight-path angle rate can be written as

$$
\dot{\gamma}=N \dot{\lambda}
$$

Integrating (5) with boundary conditions of $\gamma_{\mathrm{M}_{\mathrm{f}}}$ and $\lambda_{\mathrm{f}}$ yields

$$
\gamma_{\mathrm{M}_{\mathrm{f}}}-\gamma_{\mathrm{M}_{0}}=N\left(\lambda_{\mathrm{f}}-\lambda_{0}\right)
$$

where the subscripts 0 and $\mathrm{f}$ denote the initial and final conditions, respectively. Combining (4) and (5) to give

$$
\dot{\sigma}_{M}=\dot{\gamma}_{M}-\dot{\lambda}=(N-1) \dot{\lambda}
$$

Also integrating (7) with boundary conditions of $\sigma_{\mathrm{M}_{\mathrm{f}}}$ and $\lambda_{\mathrm{f}}$, we can obtain

$$
\sigma_{\mathrm{M}_{\mathrm{f}}}-\sigma_{\mathrm{M}_{0}}=(N-1)\left(\lambda_{\mathrm{f}}-\lambda_{0}\right)
$$

Considering the collision triangle condition, we enable the LOS angle rate to be zero to intercept a moving target. Equation (2) should satisfy the following relationship:

$$
\mathbf{V}_{\mathrm{M}} \sin \left(\gamma_{\mathrm{M}_{\mathrm{f}}}-\lambda_{\mathrm{f}}\right)=\mathbf{V}_{\mathrm{T}} \sin \lambda_{\mathrm{f}}
$$

Expanding both sides of (9), the final LOS angle which will keep the collision triangles can be expressed as (10) with some algebraic and derivations.

$$
\lambda_{\mathrm{f}}=\tan ^{-1}\left(\frac{\sin \gamma_{\mathrm{M}_{\mathrm{f}}}}{\cos \gamma_{\mathrm{M}_{\mathrm{f}}}+\eta}\right)
$$

where $\eta=\mathbf{V}_{\mathrm{T}} / \mathbf{V}_{\mathrm{M}}$ and the function of $\tan ^{-1}$ should be calculated from atan2, which is a four-quadrant inverse tangent function.

Expressing (6) with respect to $\lambda_{0}$ leads to

$$
\lambda_{0}=\lambda_{\mathrm{f}}-\frac{\gamma_{\mathrm{M}_{\mathrm{f}}}-\gamma_{\mathrm{M}_{0}}}{N}
$$

\section{Analysis for the Switched-Gain Guidance}

Consider a two-phased guidance scheme illustrated in Figure 2 , replacing $\lambda_{0}$ with $\lambda_{\mathrm{s}}$ which is the LOS angle at the specific switching instant. Substituting (4) and (10) into (11), the relationship can be expressed as

$$
\lambda_{\mathrm{s}}=\left[\tan ^{-1}\left(\frac{\sin \gamma_{\mathrm{M}_{\mathrm{f}}}}{\cos \gamma_{\mathrm{M}_{\mathrm{f}}}+\eta}\right)-\frac{\gamma_{\mathrm{M}_{\mathrm{f}}}-\sigma_{\mathrm{M}_{\mathrm{s}}}}{N_{\mathrm{F}}}\right] \frac{N_{\mathrm{F}}}{N_{\mathrm{F}}-1},
$$

where $N_{\mathrm{F}}$ and $\sigma_{\mathrm{M}_{\mathrm{s}}}$ denote the navigation gain in the final phase and the look angle at the switching instant. And we define $N_{\text {I }}$ as the navigation gain in the initial phase which will be used next. Meanwhile, (12) means that, to be able to reach a desired impact angle when attacking a moving target, the LOS angle should be a certain value at some specific instant with a desired look angle. A switching condition $\lambda_{\mathrm{s}}$ depends on the three main parameters, which are the desired look angle, the impact angle, and the navigation gain in the final phase.

Proposition 1. Considering the variation of look angle under the PN guidance in the initial phase, the look angle should satisfy the condition: $N_{\mathrm{I}}<2, \sin ^{-1} \eta<\sigma_{\mathrm{M}}<\cos ^{-1}\left(2 \eta / N_{\mathrm{I}}-2\right)$, 


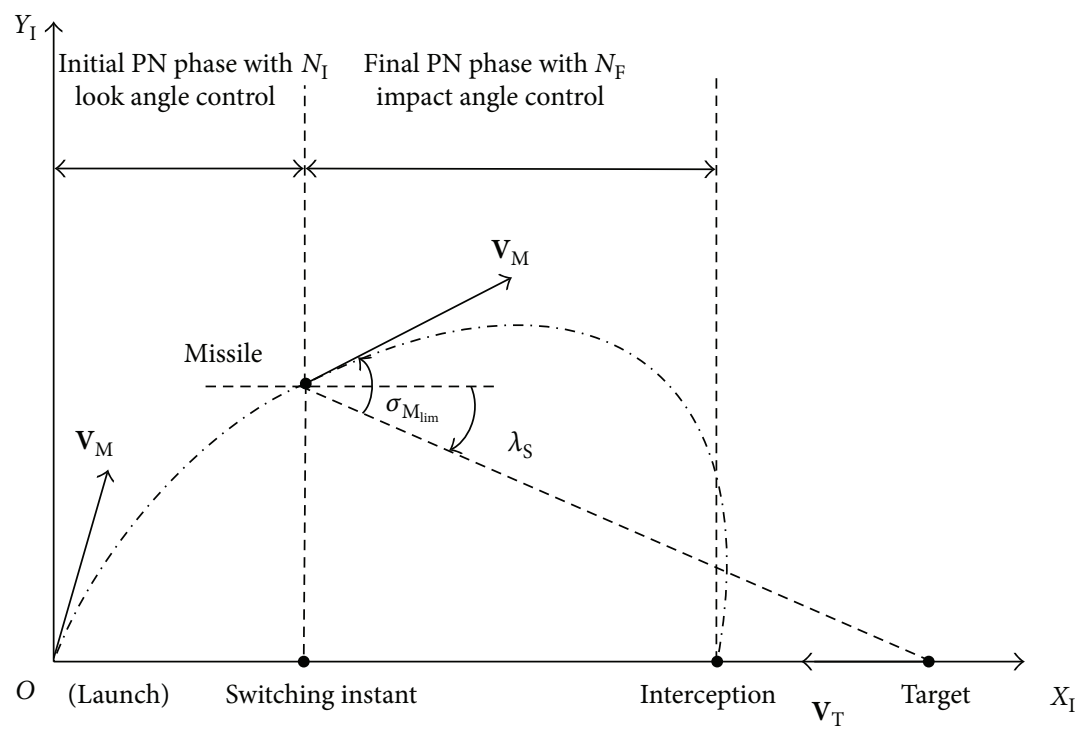

FIgURE 2: Two-phased guidance scheme.

or $N_{\mathrm{I}}>2, \sigma_{\mathrm{M}}>\max \left(\sin ^{-1} \eta, \cos ^{-1}\left(2 \eta / 2-N_{\mathrm{I}}\right)\right)$. Hence, the LOS rate $\dot{\lambda}$ with negative values strictly decreases until the switching condition $\lambda_{\mathrm{s}}$ is reached, which is $\ddot{\lambda}<0 \forall t \in\left[t_{0}, t_{\mathrm{s}}\right]$ (where $t_{\mathrm{s}}$ is the switching time of the initial phase and final phase).

Proof. Differentiating (2) with respect to $t$, we can obtain

$$
\ddot{\lambda}=\frac{\left(\mathbf{V}_{\mathrm{T}} \cos \lambda-\mathbf{V}_{\mathrm{M}} \cos \sigma_{\mathrm{M}}(N-1)-\dot{r}\right) \dot{\lambda}}{r} .
$$

Substituting (1) and (2) into (13) leads to

$\ddot{\lambda}=\frac{\mathbf{V}_{\mathrm{M}}^{2}}{r^{2}}\left[2 \eta \cos \lambda+(2-N) \cos \sigma_{\mathrm{M}}\right]\left(\eta \sin \lambda-\sin \sigma_{\mathrm{M}}\right)$.

To guarantee that the LOS angle decreases to the switching value monotonously, the LOS angle rate should keep in a negative value until switching, which is equivalent to

$$
\begin{aligned}
\dot{\lambda}\left(t_{0}\right)<0, & \\
\ddot{\lambda}(t)<0, & \\
& \forall t \in\left[t_{0}, t_{s}\right] .
\end{aligned}
$$

Substituting the initial condition into (2), we can get

$$
\dot{\lambda}\left(t_{0}\right)=-\frac{\mathbf{V}_{\mathrm{M}} \sin \sigma_{\mathrm{M}_{0}}}{r_{0}},
$$

when $\sigma_{\mathrm{M}_{0}} \in[0, \pi / 2], \dot{\lambda}\left(t_{0}\right)<0$. Since the value of $\mathbf{V}_{\mathrm{M}}^{2} / r^{2}$ must be positive to ensure $\ddot{\lambda}<0$ and $\dot{\lambda}<0$, the following conditions should be satisfied:

$$
\begin{array}{r}
2 \eta \cos \lambda+(2-N) \cos \sigma_{\mathrm{M}}>0, \\
\eta \sin \lambda-\sin \sigma_{\mathrm{M}}<0 .
\end{array}
$$

As we know that $|\sin \lambda|_{\max }=1, \quad|\cos \lambda|_{\max }=1$ for $\lambda \in[-\pi, 0]$, the inequalities in (17) can be expressed as

$$
\begin{aligned}
\sin ^{-1} \eta & <\sigma_{\mathrm{M}}<\cos ^{-1}\left(\frac{2 \eta}{N-2}\right), \quad N<2, \\
\sigma_{\mathrm{M}} & >\max \left(\sin ^{-1} \eta, \cos \left(\frac{2 \eta}{2-N}\right)\right), \quad N>2 .
\end{aligned}
$$

Replacing the navigation gain $N$ with $N_{\mathrm{I}}$ in (18), it satisfies the condition proposed in Proposition 1.

Proposition 2. For initial guidance phases, the condition of $N_{\mathrm{I}}<1$ and $\sigma_{\mathrm{M}_{0}}>\sin ^{-1} \eta, \sigma_{\mathrm{M}_{\mathrm{lm}}}<\cos ^{-1}\left(2 \eta / N_{\mathrm{I}}-2\right)$ can ensure that the look angle will increase from $\sigma_{\mathrm{M}_{0}}$ to the limit value $\sigma_{\mathrm{M}_{\mathrm{lim}}}$ during the time of $\left(t_{\mathrm{s}}-t_{0}\right)$, monotonously.

Proof. To guarantee that the look angle increases monotonously, the look angle rate $\dot{\sigma}_{\mathrm{M}}$ should be positive. As proven in Proposition $1, \dot{\lambda}<0$ when the relationship of (18) is satisfied. Combined with (7), it should meet the condition that is $N_{\mathrm{I}}<1$ to keep $\dot{\sigma}_{\mathrm{M}}>0$ with $\dot{\lambda}<0$. This condition means that

$$
\begin{aligned}
\sigma_{\mathrm{M}_{\text {min }}} & =\sigma_{\mathrm{M}_{0}}, \\
\sigma_{\mathrm{M}_{\max }} & =\sigma_{\mathrm{M}_{\mathrm{lim}}}, \\
& \forall t \in\left[t_{0}, t_{\mathrm{s}}\right] .
\end{aligned}
$$

Substituting (19) into (18) considering the monotonicity of the look angle, we can get the inequalities as

$$
\begin{gathered}
\sigma_{\mathrm{M}_{0}}>\sin ^{-1} \eta \\
\sigma_{\mathrm{M}_{\mathrm{lim}}}<\cos ^{-1}\left(\frac{2 \eta}{N_{\mathrm{I}}-2}\right) .
\end{gathered}
$$


Integrating (7) with the boundary conditions of $\sigma_{\mathrm{M}_{\mathrm{s}}}$ and $\lambda_{\mathrm{s}}$ yields

$$
\sigma_{\mathrm{M}_{\mathrm{s}}}-\sigma_{\mathrm{M}_{0}}=\left(N_{\mathrm{I}}-1\right)\left(\lambda_{\mathrm{s}}-\lambda_{0}\right)
$$

\section{Implementation of the Two-Phased Guidance Law}

To avoid violating the look angle limitation, the switching instant that look angle increases monotonically according to Proposition 2 is designed to reach the limit value exactly. According to (21), the navigation gain of initial can be expressed as

$$
N_{\mathrm{I}}=\frac{\sigma_{\mathrm{M}_{\mathrm{lim}}}-\sigma_{\mathrm{M}_{0}}}{\lambda_{\mathrm{s}}}+1
$$

In (22), $\lambda_{\mathrm{s}}$ should be calculated by (12) and actually decided by $N_{\mathrm{F}}$ with certain values of $\gamma_{\mathrm{M}_{\mathrm{f}}}$ and $\sigma_{\mathrm{M}_{\mathrm{s}}}$. Establish the proportion relation between $N_{\mathrm{F}}$ and $N_{\mathrm{I}}$, which yields

$$
k=\frac{N_{\mathrm{F}}}{N_{\mathrm{I}}} .
$$

Substituting (12) and (23) into (22) leads to

$N_{\mathrm{I}}=\frac{\sigma_{\mathrm{M}_{\mathrm{lim}}}-\sigma_{\mathrm{M}_{0}}}{\left[\tan ^{-1}\left(\sin \gamma_{\mathrm{M}_{\mathrm{f}}} / \cos \gamma_{\mathrm{M}_{\mathrm{f}}}+\eta\right)-\gamma_{\mathrm{M}_{\mathrm{f}}}-\sigma_{\mathrm{M}_{\mathrm{lim}}} / k N_{\mathrm{I}}\right]\left(k N_{\mathrm{I}} / k N_{\mathrm{I}}-1\right)}+1$.

Expanding (24), we can obtain a quadratic equation with respect to $N_{\text {I }}$ as shown in (25), where the proportionality coefficient should be regarded as a known parameter. To solve the value of unknown parameter $N_{\text {I }}$ of (25), a simple algebraic method is applied in (26).

$$
\begin{gathered}
k \lambda_{\mathrm{f}} N_{\mathrm{I}}^{2}-\left[k\left(\lambda_{\mathrm{f}}+\sigma_{\mathrm{M}_{\mathrm{lim}}}-\sigma_{\mathrm{M}_{0}}\right)+\gamma_{\mathrm{M}_{\mathrm{f}}}-\sigma_{\mathrm{M}_{\mathrm{lim}}}\right] N_{\mathrm{I}} \\
+\gamma_{\mathrm{M}_{\mathrm{f}}}-\sigma_{\mathrm{M}_{0}}=0 \\
a=\lambda_{\mathrm{f}} \\
b=-\left(k b_{1}+b_{2}\right), \\
c=\gamma_{\mathrm{M}_{\mathrm{f}}}-\sigma_{\mathrm{M}_{0}},
\end{gathered}
$$

where $b_{1}=\lambda_{\mathrm{f}}+\sigma_{\mathrm{M}_{\mathrm{lim}}}-\sigma_{\mathrm{M}_{0}}$ and $b_{2}=\gamma_{\mathrm{M}_{\mathrm{f}}}-\sigma_{\mathrm{M}_{\mathrm{lim}}}$. The solution of (25) should be

$$
N_{\mathrm{I}}=\frac{\left(k b_{1}+b_{2}\right) \pm \sqrt{b_{1}^{2} k^{2}+\left(2 b_{1} b_{2}-4 a c\right) k+b_{2}^{2}}}{2 a k},
$$

and the discriminant should be nonnegative as

$$
b_{1}^{2} k^{2}+\left(2 b_{1} b_{2}-4 a c\right) k+b_{2}^{2} \geq 0 \text {. }
$$

Therefore, to guarantee the real solution of (25), (28) should be satisfied and the inequality relation merely yields

$$
\begin{array}{r}
k \geq \frac{\left(4 a c-2 b_{1} b_{2}\right)+4 \sqrt{a c\left(a c-b_{1} b_{2}\right)}}{2 b_{1}^{2}}, \\
\text { or } k \leq \frac{\left(4 a c-2 b_{1} b_{2}\right)-4 \sqrt{a c\left(a c-b_{1} b_{2}\right)}}{2 b_{1}^{2}},
\end{array}
$$

where $a$ and $c$ should be in same sign and satisfy $a c \geq b_{1} b_{2}$. These parameters in (29) are only determined by some boundary conditions and constraints, such as $\sigma_{\mathrm{M}_{0}}, \sigma_{\mathrm{M}_{\mathrm{lim}}}$, and $\gamma_{\mathrm{M}_{\mathrm{f}}}$.

As expressed in (27), $N_{\text {I }}$ has two values that will be discussed. The one(s) less than 1 should be considered according to Proposition 2. If there is no appropriate value to satisfy Proposition 2, the proportionality coefficient $k$ needs to be chosen again. In addition, note that the guidance law for moving target requires two additional measurements or estimates of $\mathbf{V}_{\mathrm{T}}$ and $\gamma_{\mathrm{T}}$ for implementation.

In summary, the proposed method can be described in procedures as:

Step 1. Give launch conditions, the initial position, velocity, and path angle of missile and target.

Step 2. Give the desired impact angle and calculate the parameters $a, b_{1}, b_{2}, c$ according to (26), then judge if the conditions of (29) are satisfied or not.

Step 3. If satisfied, calculate the range of parameter $k$ by (29). If not, change the given launch conditions.

Step 4. Calculate $N_{\mathrm{I}}$ using (27), in which there are parameters calculated in all the steps above, $N_{\mathrm{F}}=k N_{\mathrm{I}}$.

Step 5. Confirm the switching condition $\lambda_{\mathrm{s}}$ according to $N_{\mathrm{F}}$ and (12).

The proposed guidance loop is depicted in Figure 3. The switching logic is to compare the value of $\lambda$ and $\lambda_{s}$, then to decide which navigation gain is utilized.

\section{Numerical Simulations}

To investigate the characteristics of the proposed guidance law, a number of nonlinear simulations are performed in a surface-to-surface scenario. For a given desired impact angle, a limit value of look angle and final navigation gain, the switched LOS angle conditions in (12), is applied.

In the given launch conditions of simulation, a missile has a constant speed of $200 \mathrm{~m} / \mathrm{s}$ with the initial position $\left(x_{\mathrm{M}_{0}}, y_{\mathrm{M}_{0}}\right)=(0,0) m$ and initial path angle $\gamma_{\mathrm{M}_{0}}=15^{\circ}$. A moving but nonmaneuvering target has a constant speed of $50 \mathrm{~m} / \mathrm{s}$ with the initial position $\left(x_{\mathrm{T}_{0}}, y_{\mathrm{T}_{0}}\right)=(5000,0) \mathrm{m}$ and constant path angle $\gamma_{\mathrm{T}}=180^{\circ}$. The constraint of look angle is given as $\sigma_{\mathrm{M}_{\mathrm{lim}}}=30^{\circ}$. According to the given launch 


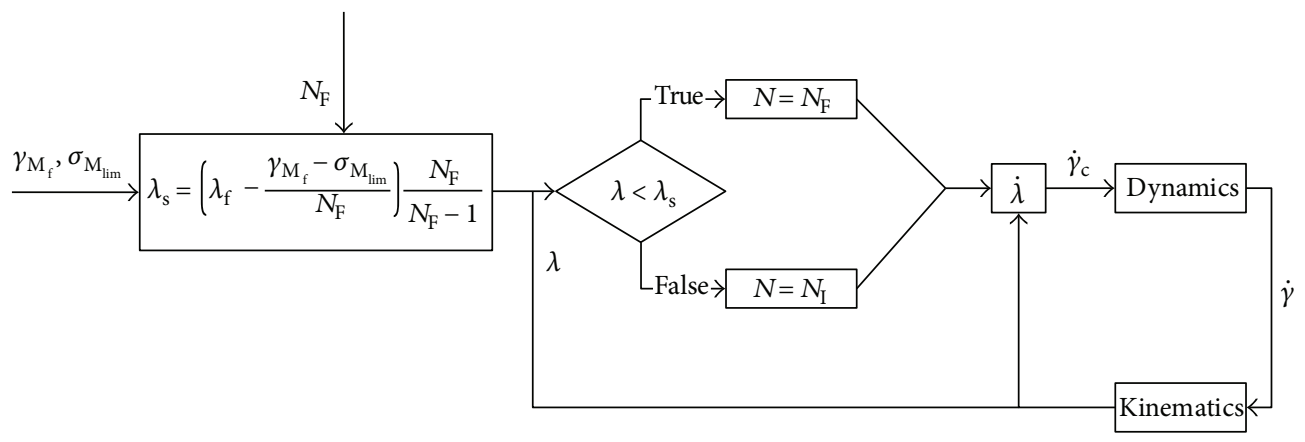

Figure 3: Switching logic of the guidance.

conditions, some important parameters and criterions for different impact angles are summarized in Table 1.

5.1. The Choice of $N_{\mathrm{I}}$ with Different $k$. From Table 1, to intercept a moving target with desired TIAC, a suitable $k$, which has two values, should be chosen to design $N_{\mathrm{I}}$ and $N_{\mathrm{F}}$. The basic principles for choosing such a value come from Proposition 2, and $N_{\mathrm{I}}<1$ should be fulfilled. The larger value of $k$ is chosen to satisfy the relation $N_{\mathrm{F}}>N_{\mathrm{I}}$ [18]. In Table 2, the values are presented where $N_{\mathrm{I}}$ is chosen based on different $k$ with specific terminal constraint $\gamma_{\mathrm{M}_{\mathrm{f}}}=-90^{\circ}$.

As shown in Table 2, all the values of $N_{\mathrm{I}}$ satisfy Proposition 2. Figure 4 displays the scenarios under the given launch conditions. In Figure 4(a), how the trajectories reach the interception point can be observed, as they have the same impact angle but using different navigation gains (both $N_{\mathrm{I}}$ and $N_{\mathrm{F}}$ ). The black dash line represents the trajectory of the moving target. Figures 4(b) and 4(c) show the variations of path angle and look angle in all scenarios. It is easy to see that each curve of path angle reaches the desired impact angle in an approaching case. Figure 4(c) shows that, under the guidance law proposed in this work, the look angles can vary in the same trend without violating the FOV limitation, while switching at different time. As calculated and shown in Table 2, 3.56 is the critical value of $k$, which leads to two solutions of $N_{\text {I }}$ that are almost the same. On the contrary, if we choose $k$ out of the critical value, it will correspond with two quite different solutions of $N_{\mathrm{I}} \cdot N_{\mathrm{F}}$ is calculated by (23), with the result that one value is larger than 2 and the other less and equal. As revealed in Figure 4(d), the scenarios of $k$ $=4, N_{\mathrm{I}}=0.49$ and $k=5, N_{\mathrm{I}}=0.37$ which simultaneously lead to $N_{\mathrm{F}}<2$ trend to make the missile's accelerations diverge. Besides, the scenarios of $k=4, N_{\mathrm{I}}=0.71$ and $k=5$, $N_{\text {I }}=0.75$ make the missile's accelerations sudden change from near zero to a much larger value. When $k=3.65$ is applied, the acceleration profile depicted in Figure 4(d) looks to be implemented easier.

5.2. Simulations of Constant Velocity Missile Model. All the simulation cases in this subsection are performed with the constant velocity missile model.

Case 1 (simulations for various impact angles). As discussed in the last subsection, the minimum value of $k$ can make a minimum abrupt acceleration change of the missile. In this
TABLE 1: Summary of the parameters and criterions.

\begin{tabular}{lccccc}
\hline$\gamma_{\mathrm{M}_{\mathrm{f}}}(\mathrm{deg})$ & $a$ & $b_{1}$ & \multicolumn{1}{c}{$b_{2}$} & $c$ & $k$ \\
\hline-50 & -0.71 & -0.45 & -1.40 & -1.13 & $k \geq 8.72$ or $k \leq 1.12$ \\
-70 & -1.01 & -0.75 & -1.75 & -1.48 & $k \geq 4.95$ or $k \leq 1.10$ \\
-90 & -1.33 & -1.06 & -2.09 & -1.83 & $k \geq 3.56$ or $k \leq 1.09$ \\
\hline
\end{tabular}

TABLe 2: Values of $N_{\text {I }}$ with different $k$.

\begin{tabular}{lc}
\hline$k$ & $N_{\mathrm{I}}$ \\
\hline 3.56 & $N_{\mathrm{I}, 1}=0.62, N_{\mathrm{I}, 2}=0.63$ \\
4.00 & $N_{\mathrm{I}, 1}=0.49, N_{\mathrm{I}, 2}=0.71$ \\
5.00 & $N_{\mathrm{I}, 1}=0.37, N_{\mathrm{I}, 2}=0.75$ \\
\hline
\end{tabular}

subsection, Figure 5 reflects the performance of the proposed guidance law with various impact angles, using the critical value of $k$ from Table 1 for every scenario. Figure 5(a) exhibits the trajectories resulting from the two-phased guidance strategy. The trajectories satisfy the different impact angle constraints and engage the curve of incoming target. Satisfying the impact angle constraint, which can be observed clearly in Figure 5(b), also influences the switching condition between the initial phase and final phase. The switching condition is obviously revealed from the trend variation of the look angle curves in Figure 5(c). It is evident that when the look angle increases to the limit, the navigation gain will switch to make the look angle decrease. Precisely because of the variation of navigation gain at switching instant, a sudden change appears in each acceleration history as shown in Figure 5(d).

Case 2 (comparative cases with other guidance laws). The performance of the proposed guidance scheme, which is illustrated in Figure 6 with the impact angle constraint of $\gamma_{\mathrm{M}_{\mathrm{f}}}=-90^{\circ}$, is compared to the BPN guidance law in [10] and a two-phased guidance scheme described in [28]. The implemented acceleration in [28] is summarized as

$$
\mathbf{a}_{\mathrm{M}}= \begin{cases}\mathbf{V}_{\mathrm{M}} \dot{\lambda}+K\left(\sigma_{\mathrm{d}}-\sigma\right), & \text { for }|\lambda|<\left|\lambda_{s}\right| \\ N \mathbf{V}_{\mathrm{M}} \dot{\lambda}, & \text { for }|\lambda| \geq\left|\lambda_{s}\right| .\end{cases}
$$




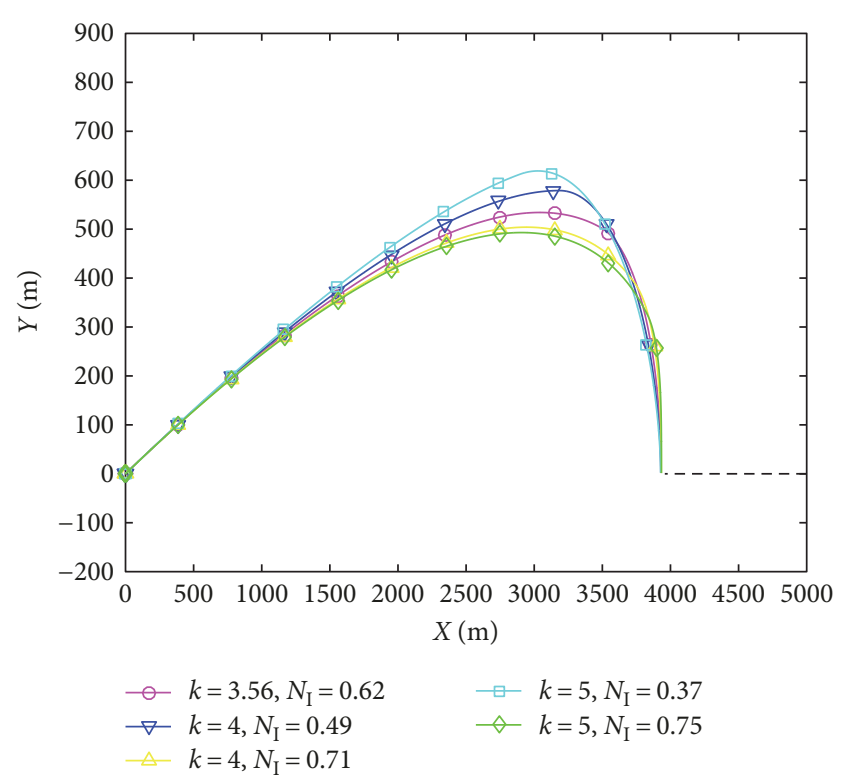

(a) Engagement trajectories

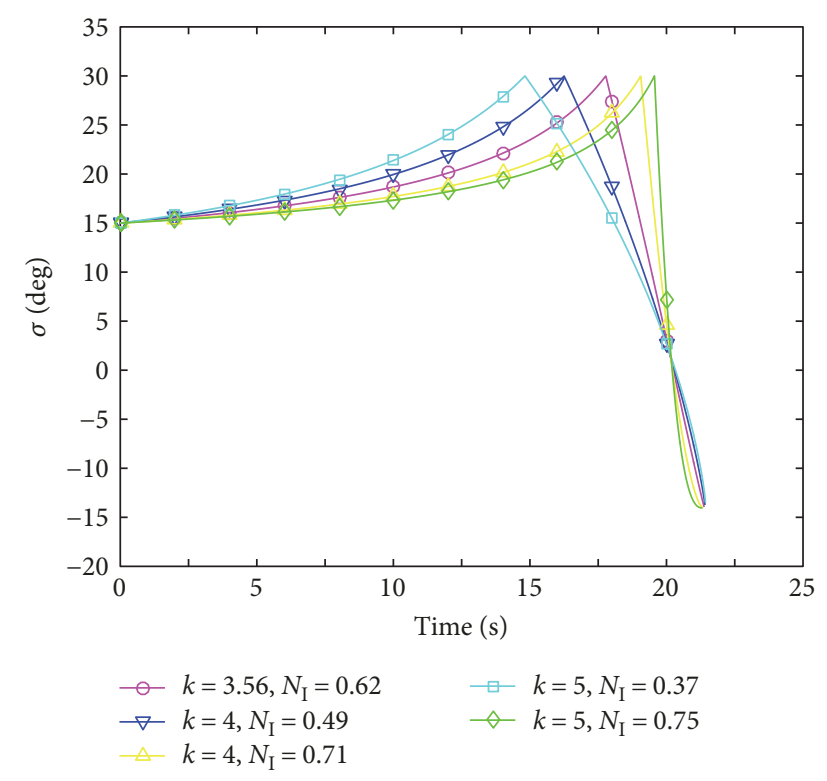

(c) Look angles

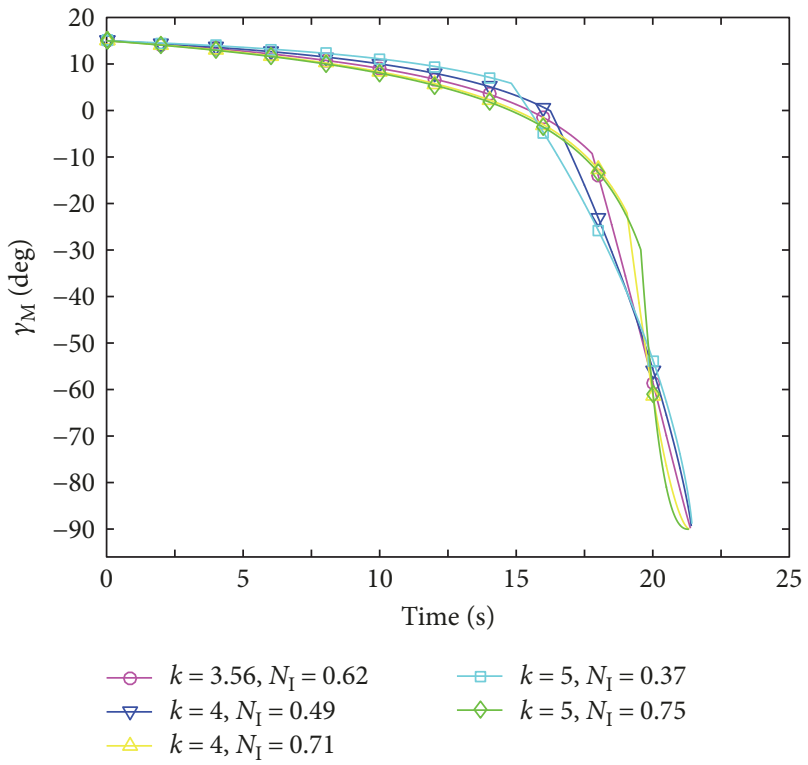

(b) Flight-path angles

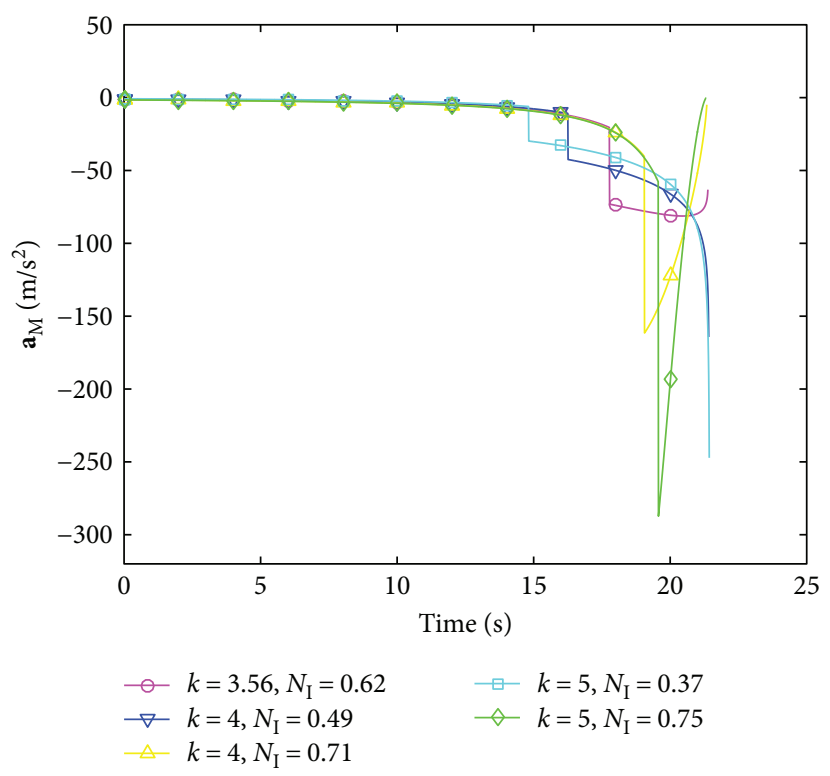

(d) Acceleration histories

FIGURE 4: The results for the different choice of $N_{\mathrm{I}}$ with $\gamma_{\mathrm{M}_{\mathrm{f}}}=-90^{\circ}$.

In Figure 6(a), it is obvious that the trajectories of BPN and [28] appear more curved than the proposed one. For the terminal impact constraint, all the methods can satisfy the demand as shown in Figure 6(b). However, the look angle variations are quite distinct from each other as seen in Figure 6(c). The BPN guidance law violates the seeker's FOV limitation at a certain time. The method in [28] leads the look angle increase to the limit rapidly and then maintain at this value until switching. Similarly, the method presented in this work controls the look angle to increase to the limit, but slowly and monotonously until switching. In Figure 6(d), compared with the scheme in [28], the acceleration history produced by the proposed method appears more smoothly especially at initial time.

Case 3 (comparative cases considering first-order lag). Several simulation cases for the guidance law in [28] and the proposed one in this work are carried out to compare the performance with regard to autopilot delay. Consider a first-order lag system in this case, which can be described with a time constant $T_{\mathrm{m}}$

$$
\frac{\mathbf{a}_{\mathrm{M}}}{\mathbf{a}_{\mathrm{C}}}=\frac{1}{T_{\mathrm{m}} s+1},
$$



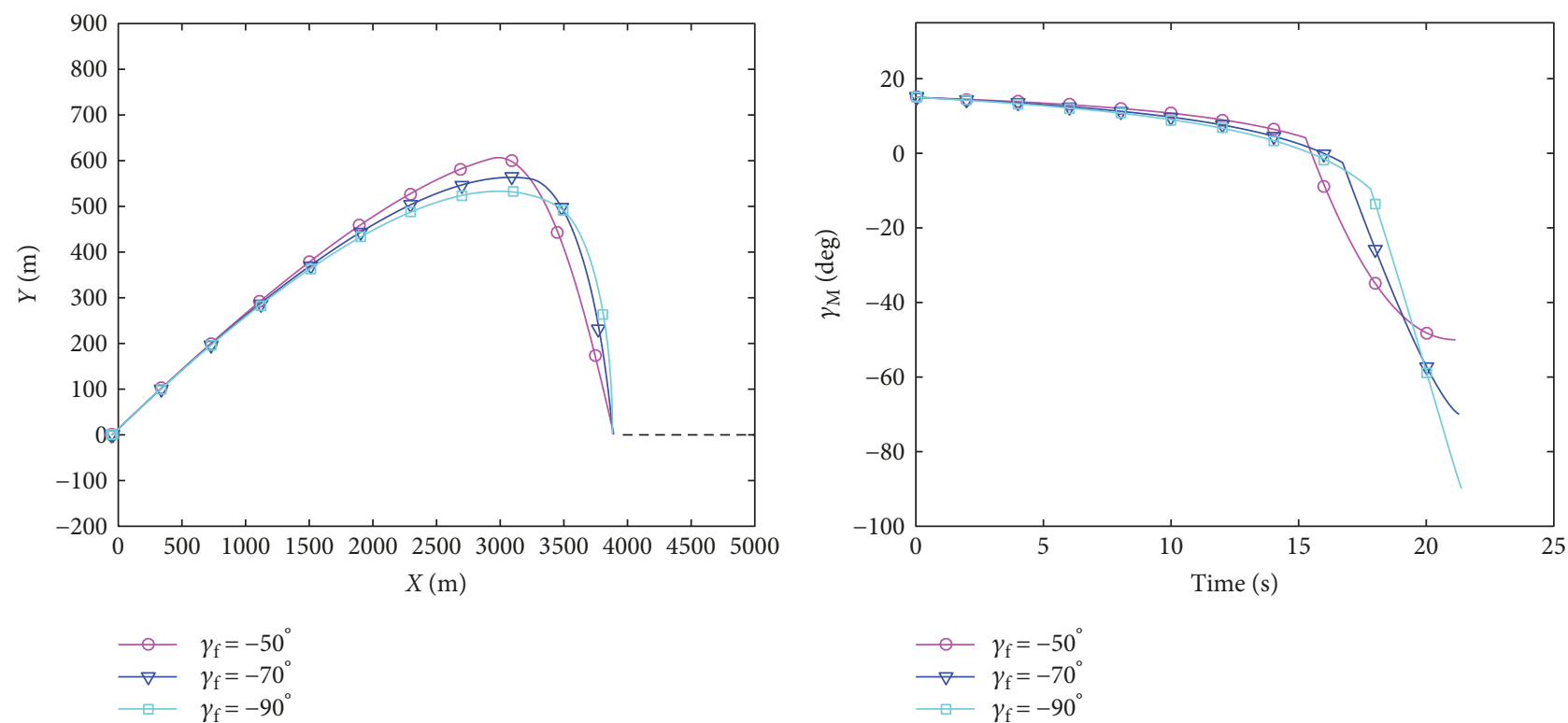

(a) Engagement trajectories

(b) Flight-path angles

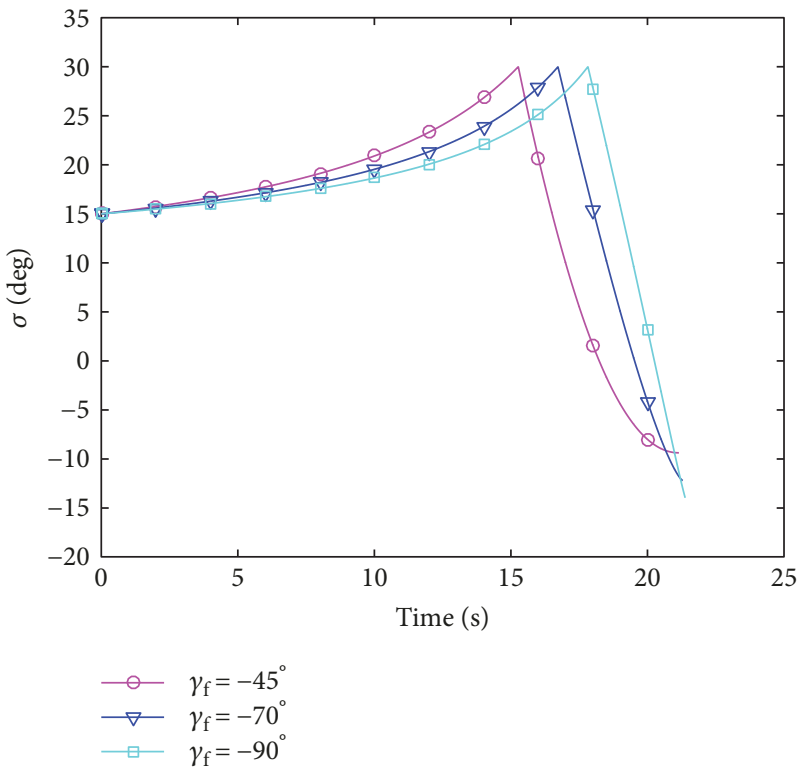

(c) Look angles

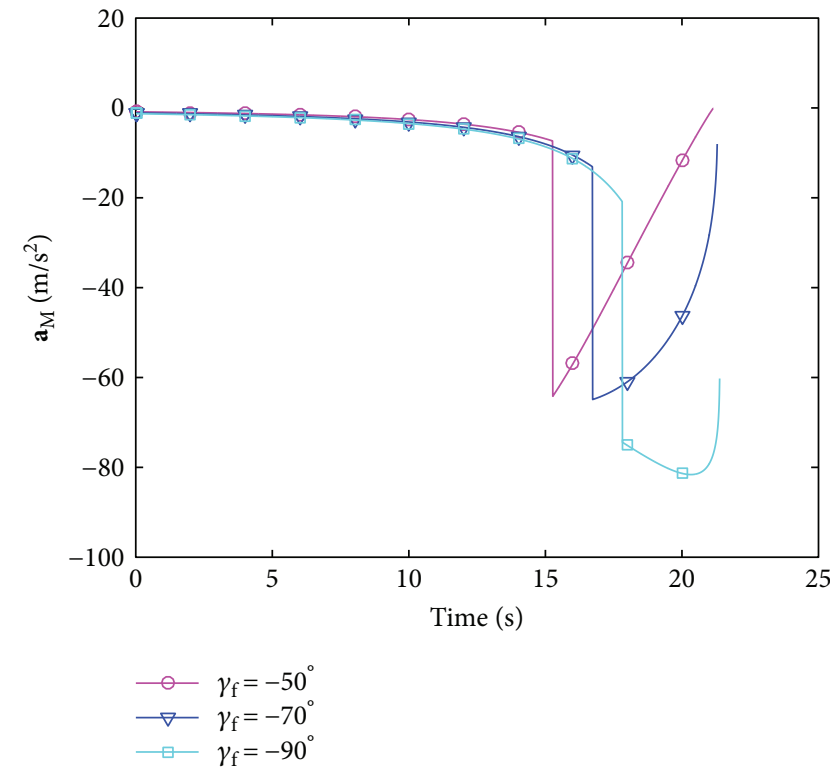

(d) Acceleration histories

FIgURE 5: The results for the different terminal impact angles.

where $\mathbf{a}_{\mathrm{M}}$ and $\mathbf{a}_{\mathrm{C}}$ represent the lagged system response and the acceleration command, respectively.

To observe the autopilot delay influence on the guidance accuracy, $T_{\mathrm{m}}$ is set to vary from 0 to 0.3 with the TIAC of $-70^{\circ}$. Comparative results of $T_{\mathrm{m}}=0.25$ are illustrated in Figure 7. When the autopilot lag is considered, the differences between Figures 6(c) and 7(b) show that the look angle of [17] exceeds the FOV limit at first in the initial phase. The impact angle error (IAE) is described in Figure 8 with the variation of $T_{\mathrm{m}}$. The IAE of the proposed guidance law in this work is less than that of [28], for each investigated value of $T_{\mathrm{m}}$. The different trends of the two curves shown in Figure 8 indicate that, compared with [28], the influence on the proposed method varies not obviously with the increasing of $T_{\mathrm{m}}$. The IAE of the proposed guidance law can keep in a small value.

5.3. Simulations of Realistic Missile Model with Uncertain Target Velocity. In this subsection, further simulations are carried out with a realistic missile model considering the uncertainty of target's velocity.

Case 4 (realistic model with uncertainty cases). The aerodynamics drag and gravity are taken into account in the realistic 


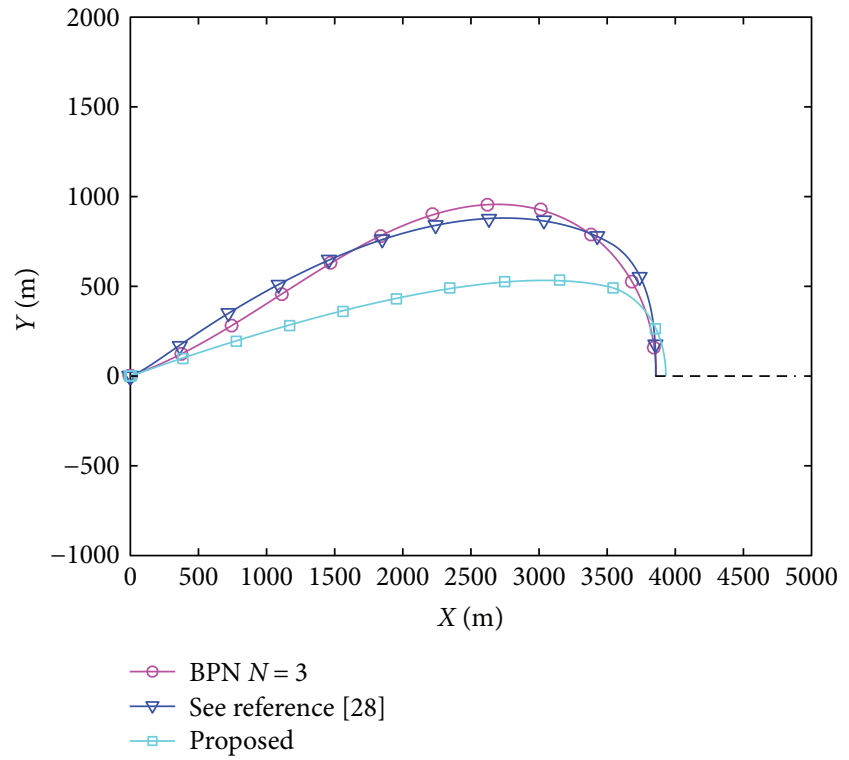

(a) Engagement trajectories

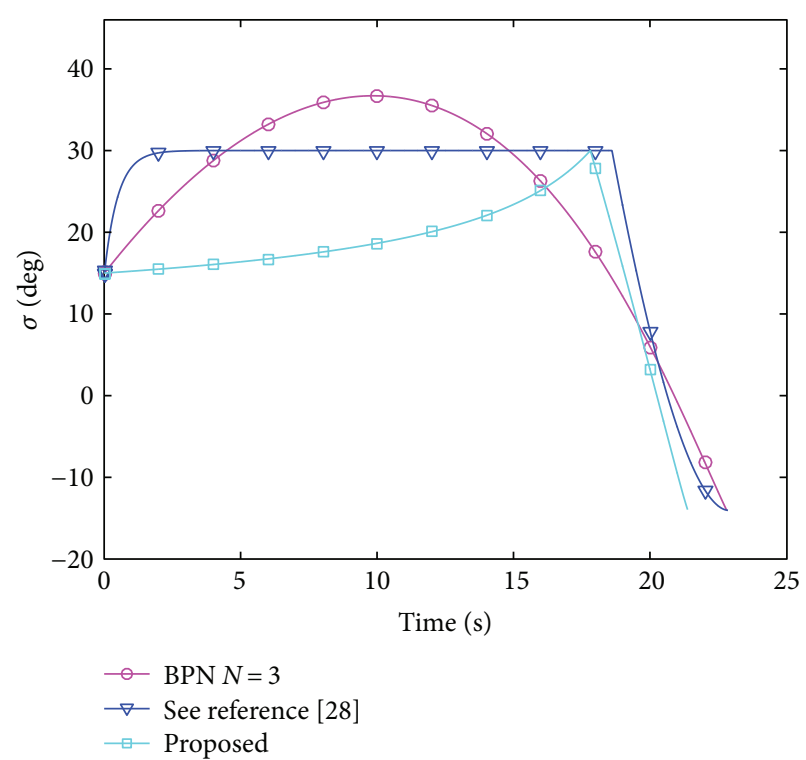

(c) Look angles

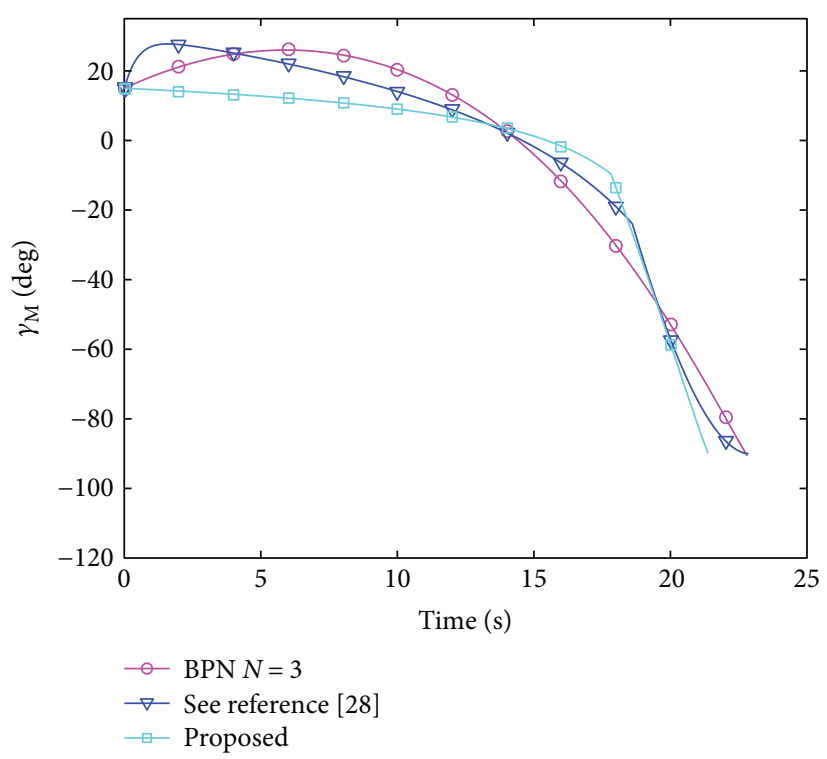

(b) Flight-path angles

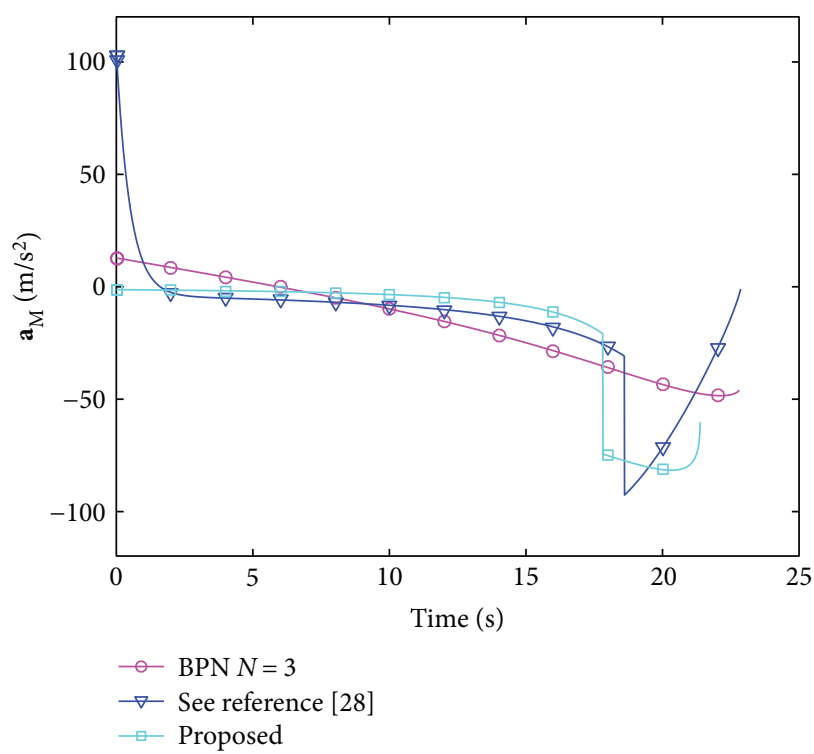

(d) Acceleration histories

FIGURE 6: The results for the comparative cases with $\gamma_{\mathrm{M}_{\mathrm{f}}}=-90^{\circ}$.

missile model, which is taken from [30]. The nonlinear dynamics equations are given by

$$
\begin{aligned}
& \dot{x}_{\mathrm{M}}=\mathbf{V}_{\mathrm{M}} \cos \gamma_{\mathrm{M}}, \\
& \dot{y}_{\mathrm{M}}=\mathbf{V}_{\mathrm{M}} \sin \gamma_{\mathrm{M}}, \\
& \dot{\mathbf{V}}_{\mathrm{M}}=\frac{T_{\mathrm{M}}-D_{\mathrm{M}}}{m}-g \sin \gamma_{\mathrm{M}}, \\
& \dot{\gamma}_{\mathrm{M}}=\frac{\mathbf{a}_{\mathrm{M}}-g \cos \gamma_{\mathrm{M}}}{\mathbf{V}_{\mathrm{M}}} .
\end{aligned}
$$

Without loss of generality, we assume that $T_{M}=0$ in the terminal engagement case. Furthermore, the gravity compensation term should be added to the original acceleration command, which is based on the five-step procedure proposed in this work, to offset the gravity influence on the flight-path angle rate. For realistic implementation, the original acceleration command needs to be changed as

$$
\mathbf{a}_{\mathrm{M}}=\mathbf{a}_{\mathrm{M}}^{*}+g \cos \gamma_{\mathrm{M}} \text {, }
$$

where $\mathbf{a}_{\mathrm{M}}^{*}$ represents the acceleration command for constant speed missile model. The target's velocity uncertainty is also considered in the simulations. The constant target speed $50 \mathrm{~m} / \mathrm{s}$ is regarded as a nominal value to design the proposed guidance law; however, the simulations are carried out by the percentage perturbation values which are disturbed by $20 \%$ (positive and negative) deviation about the nominal value. 


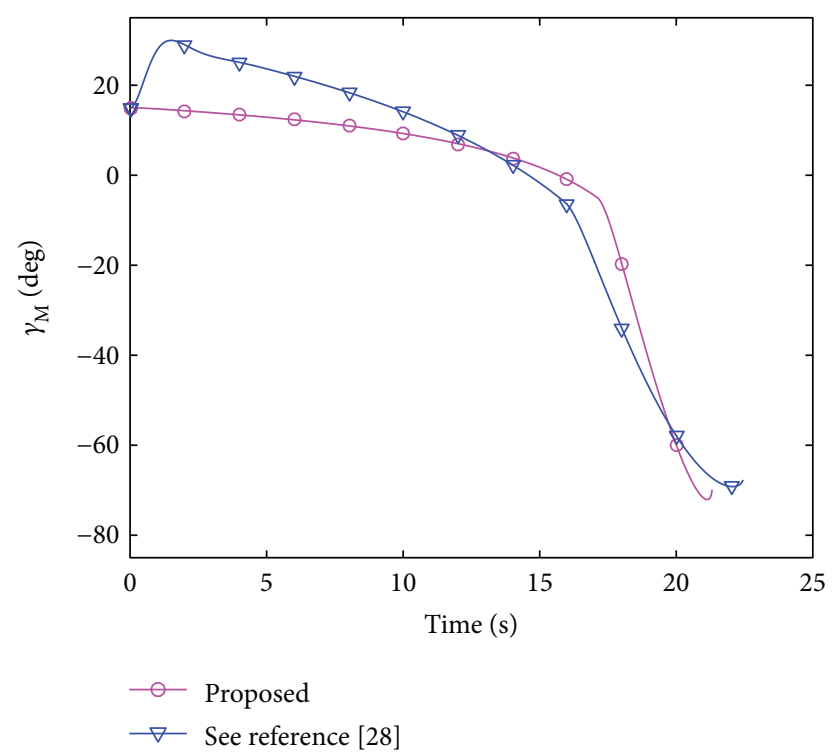

(a) Flight-path angles

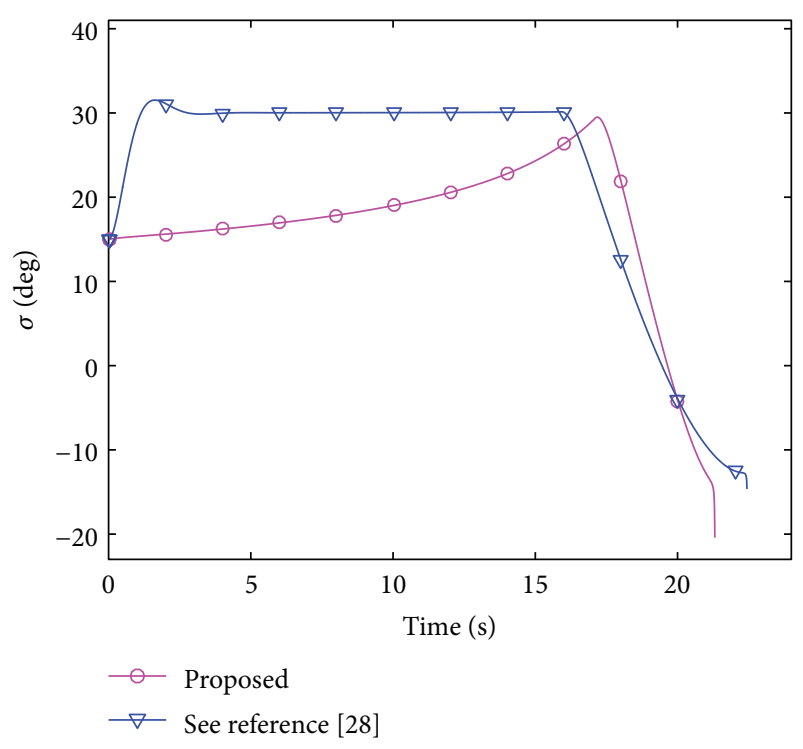

(b) Look angles

FIGURE 7: Comparative results with system lag $T_{\mathrm{m}}=0.25$ and $\gamma_{\mathrm{M}_{\mathrm{f}}}=-70^{\circ}$.

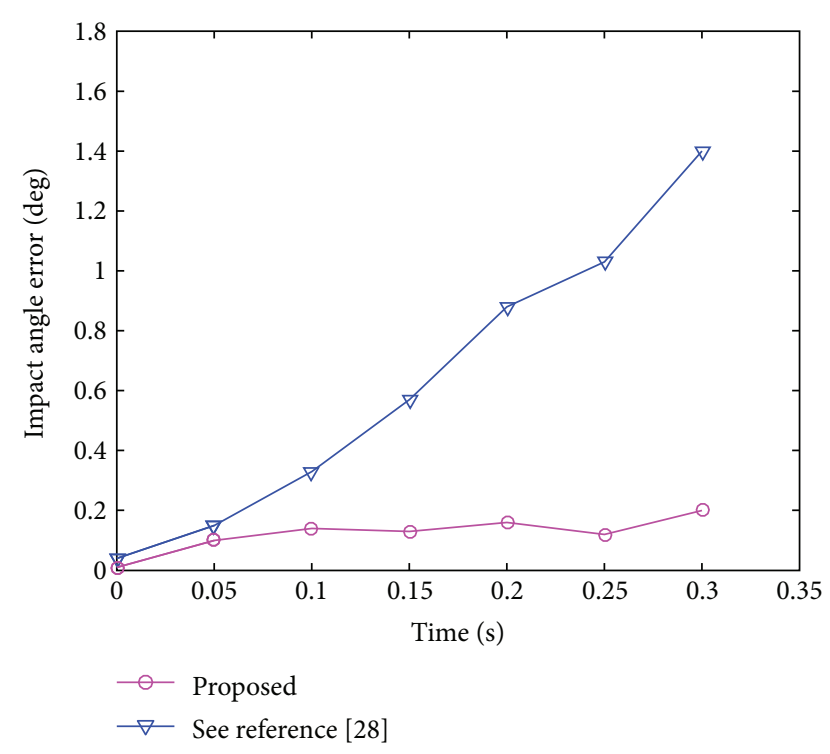

Figure 8: Impact angle error with the variation of system lag.

The detailed simulation results are displayed in Table 3 and Figure 9.

Figure 9(a) shows that the missile can successfully intercept the target with realistic missile model under the condition of uncertain target velocities. In Figure 9(b), the missile velocities vary due to the gravity and air drag. Both the nominal and disturbed target velocity cases are simulated and compared. The main influences on LOS angles, flightpath angles, and look angles are exhibited in Figures 9(c)9(e), respectively. The detailed numerical results are listed in Table 3 for comparison. Both the realistic decreasing velocity of missile and the uncertain target velocity bring the terminal impact angle errors (TIAE) in engagement simulations. The influence of target's $-20 \%$ deviation of velocity
TABLE 3: Angle constraints of different conditions.

\begin{tabular}{lcc}
\hline & TIAE (deg) & $\begin{array}{c}\text { Look angle at switching } \\
\text { instant (deg) }\end{array}$ \\
\hline$\gamma_{\mathrm{f}}=-50^{\circ}$ nominal & 4.82 & 27.35 \\
$\gamma_{\mathrm{f}}=-50^{\circ}+20 \%$ deviation & 0.82 & 27.08 \\
$\gamma_{\mathrm{f}}=-50^{\circ}-20 \%$ deviation & 8.68 & 27.41 \\
$\gamma_{\mathrm{f}}=-70^{\circ}$ nominal & 4.70 & 23.29 \\
$\gamma_{\mathrm{f}}=-70^{\circ}+20 \%$ deviation & -1.24 & 23.10 \\
$\gamma_{\mathrm{f}}=-70^{\circ}-20 \%$ deviation & 9.43 & 20.87 \\
$\gamma_{\mathrm{f}}=-90^{\circ}$ nominal & 4.67 & 29.13 \\
$\gamma_{\mathrm{f}}=-90^{\circ}+20 \%$ deviation & -2.33 & 29.25 \\
$\gamma_{\mathrm{f}}=-90^{\circ}-20 \%$ deviation & 8.12 & 22.74 \\
\hline
\end{tabular}

is added on that of decreasing missile velocity, which leads to a larger TIAE. On the contrary, the target's $+20 \%$ deviation of velocity can partly offset the influence of decreasing missile velocity, which can be observed for all the impact angles in Table 3 . The decreasing missile velocity and uncertain target velocity also effect the look angles. The look angle at switching instant is not equal to the limit FOV angle but does not exceed the limitation yet. The acceleration histories are plotted in Figure 9(f). It is clear that high impact angle constraint needs large maximum acceleration effect on the missile especially for the realistic missile model.

\section{Conclusions}

The look angle control strategy introduced in this study is motivated by the physical limit of the missile's seeker. It leads to a convenient design approach similar to the PN guidance law but taking the FOV limitation into consideration. The proposed two-phased scheme admits the allowed look angle 

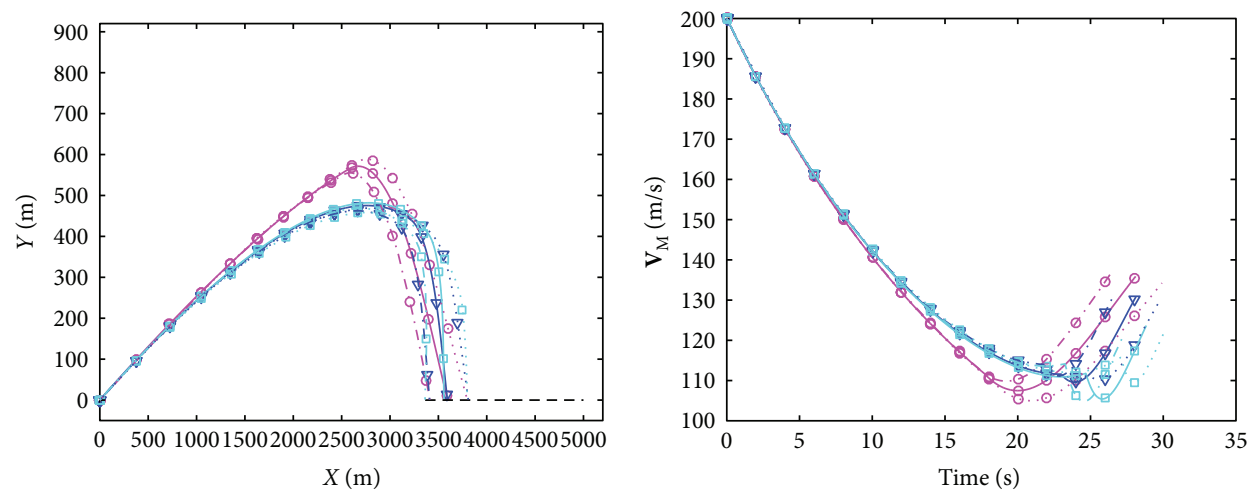

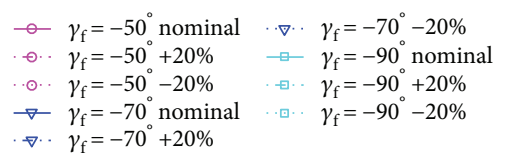

(a) Engagement trajectories

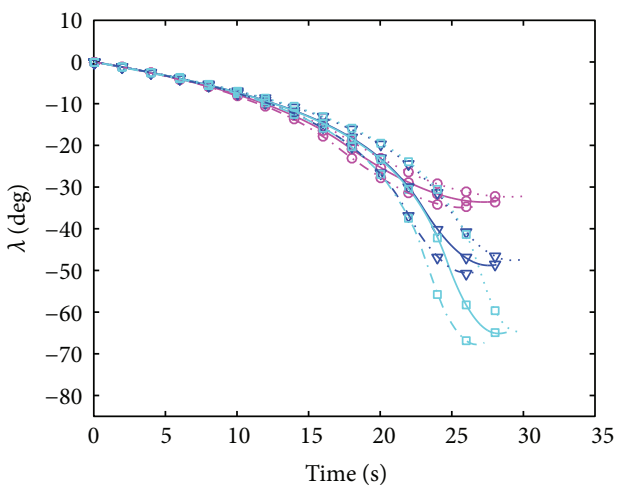

- $\gamma_{\mathrm{f}}=-50^{\circ}$ nominal $\cdot \nabla \cdot \gamma_{\mathrm{f}}=-70^{\circ}-20 \%$

๑. $\gamma_{\mathrm{f}}=-50^{\circ}+20 \% \square \gamma_{\mathrm{f}}=-90^{\circ}$ nominal

๑. $\gamma_{\mathrm{f}}=-50-20 \% \quad$ 曰. $\gamma_{\mathrm{f}}=-90+20 \%$

$\nabla \gamma_{\mathrm{f}}=-70^{\circ}$ nominal ... $\gamma_{\mathrm{f}}=-90^{\circ}-20 \%$

$\nabla$. $\gamma_{\mathrm{f}}=-70^{\circ}+20 \%$

(c) Line-of-sight angles

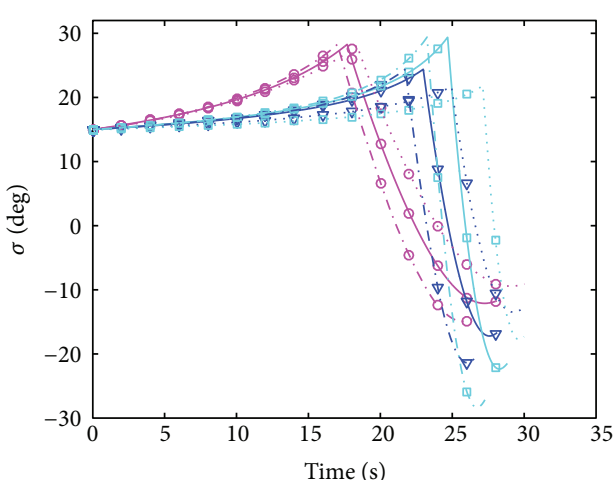

$$
\begin{array}{llll}
\odot & \gamma_{\mathrm{f}}=-50^{\circ} \text { nominal } & \nabla \cdot & \gamma_{\mathrm{f}}=-70^{\circ}-20 \% \\
\odot & \gamma_{\mathrm{f}}=-50^{\circ}+20 \% & \square & \gamma_{\mathrm{f}}=-90^{\circ} \text { nominal } \\
\odot & \gamma_{\mathrm{f}}=-50^{\circ}-20 \% & \gamma_{\mathrm{f}}=-90^{\circ}+20 \% \\
\nabla & \gamma_{\mathrm{f}}=-70^{\circ} \text { nominal } & & \gamma_{\mathrm{f}}=-90^{\circ}-20 \% \\
\nabla \cdot \gamma_{\mathrm{f}}=-70^{\circ}+20 \% & &
\end{array}
$$

(e) Look angles

$$
\begin{array}{llll}
\multimap & \gamma_{\mathrm{f}}=-50^{\circ} \text { nominal } & \cdots \nabla & \gamma_{\mathrm{f}}=-70^{\circ}-20 \% \\
\bullet & \gamma_{\mathrm{f}}=-50^{\circ}+20 \% & - & \gamma_{\mathrm{f}}=-90^{\circ} \text { nominal } \\
\odot \odot & \gamma_{\mathrm{f}}=-50^{\circ}-20 \% & \ddots & \gamma_{\mathrm{f}}=-90^{\circ}+20 \% \\
\rightarrow & \gamma_{\mathrm{f}}=-70^{\circ} \text { nominal } & \cdots & \gamma_{\mathrm{f}}=-90^{\circ}-20 \% \\
\nabla & \gamma_{\mathrm{f}}=-70^{\circ}+20 \% & &
\end{array}
$$

(b) Missile velocities

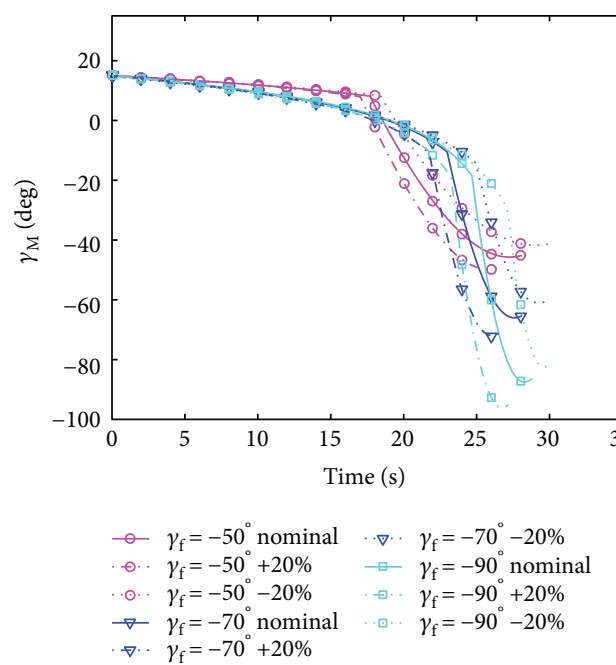

(d) Flight-path angles

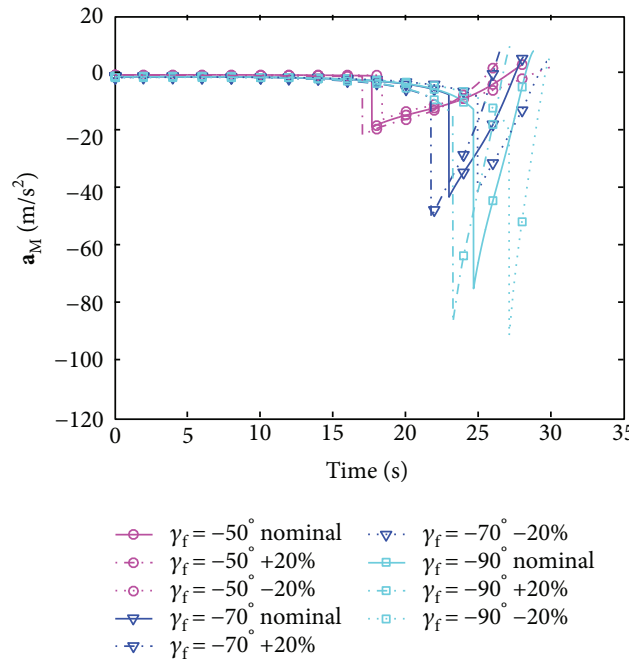

(f) Acceleration histories

Figure 9: The results for realistic missile model. 
along with the desired impact angle constraint and switches the proportional navigation gain at an appropriate instant in the case for engagement. In this article, to evaluate the specific navigation gains for the initial and final phases, the scaling factor between them is discussed. A quadratic equation regarding to the navigation gain of the initial phase is presented and solved with consideration of the minimum jump amplitude at the switching instant. Actually, the abrupt acceleration change cannot be eliminated completely because of the switching of navigation gain at a certain instant. In spite of the acceleration histories with abrupt change, this proposed method also has a simple and feasible structure for implementation because only the LOS and LOS rate information are required to shape the trajectory and decide when to switch. Simulation results indicate the performance and feasibility of the proposed guidance strategy, in terms of satisfying the terminal impact angle constraint and FOV limit. In addition, under the lag system, the proposed method can also provide a satisfactory guidance performance for TIAC without exceeding the FOV limit. When applying the proposed guidance law, the decreasing missile velocity and uncertainties in the target speed may result in some unavoidable TIAEs compared to the ideal missile and target.

\section{Conflicts of Interest}

The authors declare that there is no conflict of interests regarding the publication of this paper.

\section{Acknowledgments}

This work is supported by the National Natural Science Foundation of China (Grant no. 11202024).

\section{References}

[1] M. Kim and K. V. Grider, "Terminal guidance for impact attitude angle constrained flight trajectories," IEEE Transactions on Aerospace and Electronic Systems, vol. AES-9, no. 6, pp. 852-859, 1973.

[2] P. L. Vergez and J. R. Mcclendon, "Optimal control and estimation for strapdown seeker guidance of tactical missiles," Journal of Guidance, Control, and Dynamics, vol. 5, no. 3, pp. 225-226, 1982.

[3] R. K. Mehra and R. D. Ehrich, "Air-to-air missile guidance for strapdown seekers," in The 23rd IEEE Conference on Decision and Control, pp. 1109-1115, Las Vegas, NV, USA, 2007, IEEE.

[4] A. E. Bryson, Applied Optimal Control: Optimization, Estimation, and Control, CRC Press, 1975.

[5] P. Zarchan, Tactical and Strategic Missile Guidance, American Institute of Aeronautics \& Astronautics Inc, Washington, DC, USA, Sixth edition, 1990.

[6] C.-K. Ryoo, H. Cho, and M.-J. Tahk, "Time-to-go weighted optimal guidance with impact angle constraints," IEEE Transactions on Control Systems Technology, vol. 14, no. 3, pp. 483492, 2006.

[7] C. H. Lee, M. J. Tahk, and J. I. Lee, "Generalized formulation of weighted optimal guidance laws with impact angle constraint," IEEE Transactions on Aerospace and Electronic Systems, vol. 49, no. 2, pp. 1317-1322, 2013.
[8] C. H. Lee, T.-H. Kim, M.-J. Tahk, and I.-H. Whang, "Polynomial guidance laws considering terminal impact angle and acceleration constraints," IEEE Transactions on Aerospace and Electronic Systems, vol. 49, no. 1, pp. 74-92, 2013.

[9] N. A. Shneydor, Missile Guidance and Pursuit: Kinematics, Dynamics and Control, Elsevier, 1998.

[10] B. S. Kim, J. G. Lee, and H. S. Han, "Biased PNG law for impact with angular constraint," IEEE Transactions on Aerospace and Electronic Systems, vol. 34, no. 1, pp. 277-288, 1998.

[11] P. Lu, D. B. Doman, and J. D. Schierman, "Adaptive terminal guidance for hypervelocity impact in specified direction," Journal of Guidance, Control, and Dynamics, vol. 29, no. 2, pp. $269-278,2006$.

[12] A. Ratnoo and D. Ghose, "Impact angle constrained interception of stationary targets," Journal of Guidance, Control, and Dynamics, vol. 31, no. 6, pp. 1817-1822, 2008.

[13] A. Ratnoo and D. Ghose, "Impact angle constrained guidance against nonstationary nonmaneuvering targets," Journal of Guidance, Control, and Dynamics, vol. 33, no. 1, pp. 269275, 2010.

[14] Y. Zhang, M. Sun, and Z. Chen, "Finite-time convergent guidance law with impact angle constraint based on slidingmode control," Nonlinear Dynamics, vol. 70, no. 1, pp. 619625, 2012.

[15] Y. Zhao, Y. Sheng, and X. Liu, "Sliding mode control based guidance law with impact angle constraint," Chinese Journal of Aeronautics, vol. 27, no. 1, pp. 145-152, 2014.

[16] L. Sun, W. Wang, R. Yi, and S. Xiong, "A novel guidance law using fast terminal sliding mode control with impact angle constraints," ISA Transactions, vol. 64, pp. 12-23, 2016.

[17] S. Lyu, Z. H. Zhu, S. Tang, and X. Yan, "Prescribed performance slide mode guidance law with terminal line-of-sight angle constraint against maneuvering targets," Nonlinear Dynamics, vol. 88, no. 3, pp. 2101-2110, 2017.

[18] B. G. Park, T. H. Kim, and M. J. Tahk, "Optimal impact angle control guidance law considering the seeker's field-of-view limits," Proceedings of the Institution of Mechanical Engineers, Part G: Journal of Aerospace Engineering, vol. 227, no. 8, pp. 1347-1364, 2012.

[19] K. S. Erer and O. Merttopçuoglu, "Indirect impact-anglecontrol against stationary targets using biased pure proportional navigation," Journal of Guidance, Control, and Dynamics, vol. 35, no. 2, pp. 700-704, 2012.

[20] T. H. Kim, B. G. Park, and M. J. Tahk, "Bias-shaping method for biased proportional navigation with terminal-angle constraint," Journal of Guidance, Control, and Dynamics, vol. 36, no. 6, pp. 1810-1816, 2013.

[21] K. S. Erer, R. Tekin, and M. K. Ozgoren, "Look angle constrained impact angle control based on proportional navigation," in AIAA Guidance, Navigation, and Control Conference, pp. 91-97, Kissimmee, FL, USA, 2015.

[22] Z. Yang, H. Wang, and D. Lin, “Time-varying biased proportional guidance with seeker's field-of-view limit," International Journal of Aerospace Engineering, vol. 2016, Article ID 9272019, 11 pages, 2016.

[23] R. Tekin and K. S. Erer, "Switched-gain guidance for impact angle control under physical constraints," Journal of Guidance, Control, and Dynamics, vol. 38, no. 2, pp. 205-216, 2015.

[24] A. Ratnoo, "Analysis of two-stage proportional navigation with heading constraints," Journal of Guidance, Control, and Dynamics, vol. 39, no. 1, pp. 156-164, 2016. 
[25] Q. Wen, Q. Xia, and S. Weixia, “A parameter design strategy for seeker's field-of-view constraint in impact angle guidance," Proceedings of the Institution of Mechanical Engineers, Part G: Journal of Aerospace Engineering, vol. 229, no. 13, pp. 23892396, 2015.

[26] S. He and D. Lin, "A robust impact angle constraint guidance law with seeker's field-of-view limit," Transactions of the Institute of Measurement and Control, vol. 37, no. 3, pp. 317-328, 2015.

[27] X. Wang, Y. Zhang, and H. Wu, "Sliding mode control based impact angle control guidance considering the seeker's fieldof-view constraint," ISA Transactions, vol. 61, pp. 49-59, 2016.

[28] B. G. Park, H.-H. Kwon, Y.-H. Kim, and T.-H. Kim, "Composite guidance scheme for impact angle control against a nonmaneuvering moving target," Journal of Guidance, Control, and Dynamics, vol. 39, no. 5, pp. 1132-1139, 2016.

[29] B. G. Park, T. H. Kim, and M. J. Tahk, "Biased PNG with terminal-angle constraint for intercepting nonmaneuvering targets under physical constraints," IEEE Transactions on Aerospace and Electronic Systems, vol. 53, no. 3, pp. 15621572, 2017.

[30] P. Kee, D. Li, and S. Chai, "Near optimal midcourse guidance law for flight vehicle," in 36th AIAA Aerospace Sciences Meeting and Exhibit, pp. 583-590, Reno, NV, USA, 2006. 


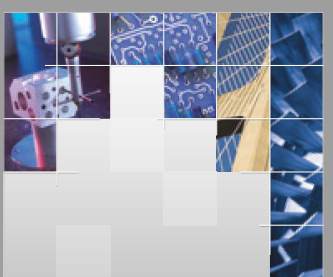

\section{Enfincering}
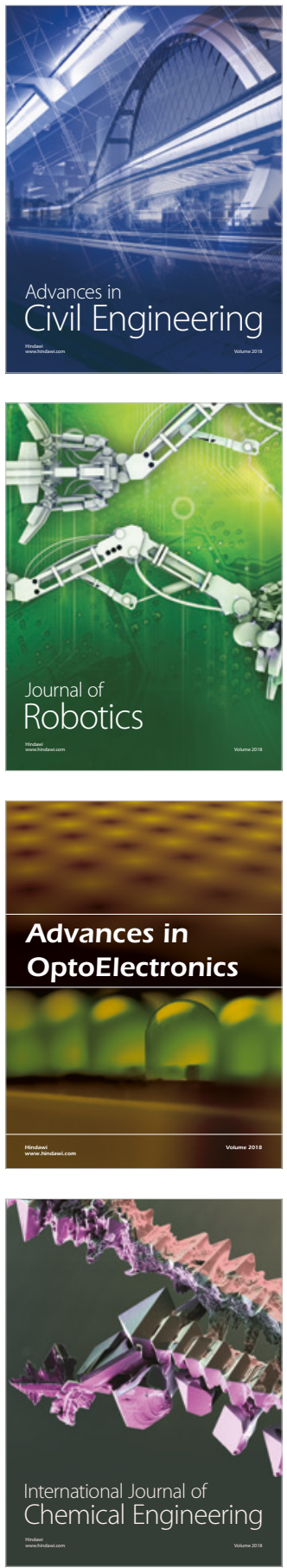

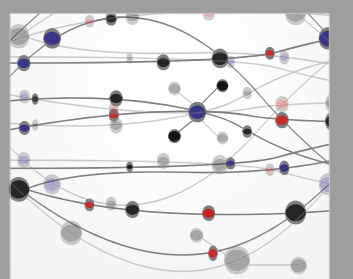

\section{Rotating \\ Machinery}

The Scientific World Journal

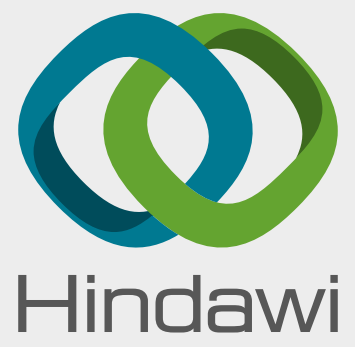

Submit your manuscripts at

www.hindawi.com
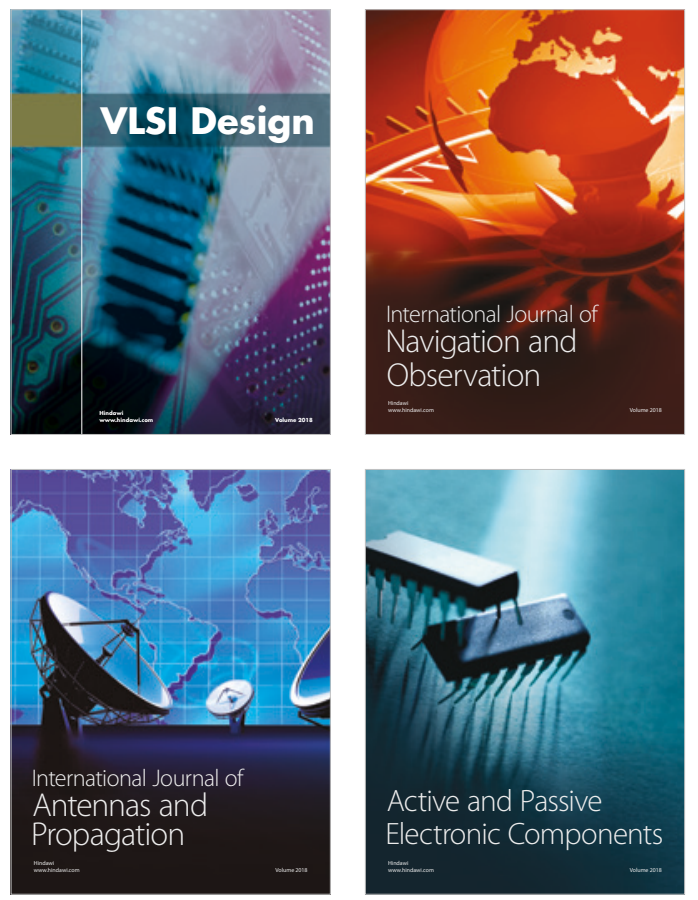
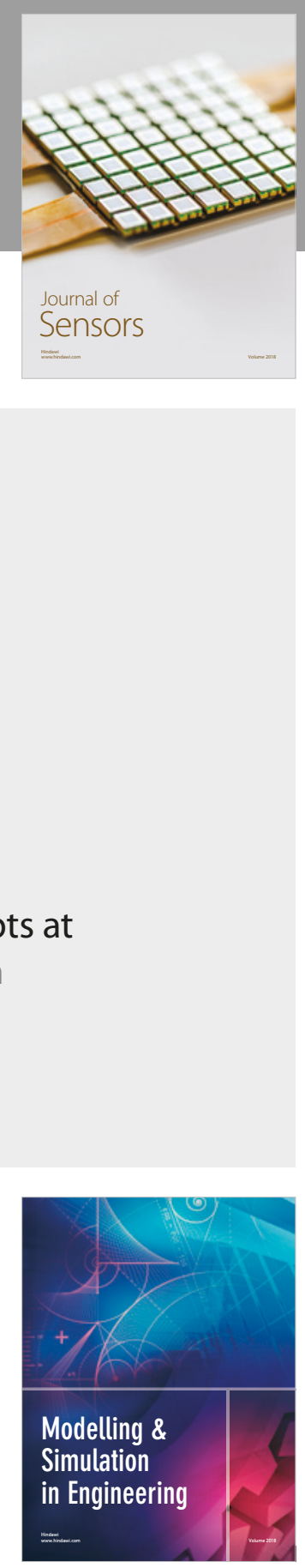

\section{Advances \\ Multimedia}
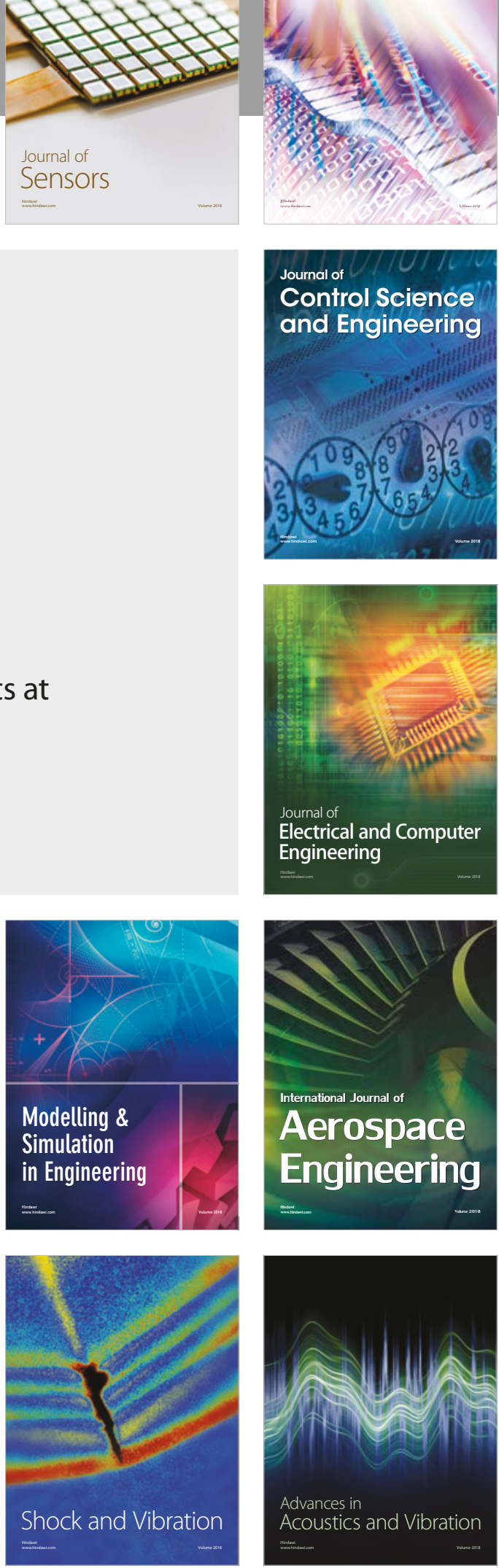